\title{
Rootstock and Training System Affect Dry-matter and Carbohydrate Distribution in 'Golden Delicious' Apple Trees
}

\author{
Gary W. Stutte \\ Bionetics Corporation, Mail Code-Bio 3, Kennedy Space Center, FL 32899 \\ Tara A. Baugher, Sandra P. Walter, and David W. Leach \\ West Virginia University Plant and Soil Sciences Experiment Farm, Kearneysville, WV 25430 \\ D. Michael Glenn and Thomas J. Tworkoski \\ Appalachian Fruit Research Station, U.S. Department of Agriculture, Agricultural Research Service, \\ Kearneysville, WV 25430 \\ Additional index words. Malus domestica, mobilizable carbohydrates, starch, sorbitol, glucose, fructose, sucrose, vigor,
pruning
}

\begin{abstract}
A study was conducted to quantify the effects of rootstock and training system on C allocation in apple. Drymatter distribution was determined at harvest in 5-year-old 'Golden Delicious' apple (Malus domestica Borkh.) trees on four rootstocks (MM.111 EMLA, M.7a, M.26 EMLA, and M.9 EMLA) and in three training systems (three-wire palmette, free-standing central leader, and nonpruned). Mobilizable carbohydrate content was determined at harvest and leaf fall in trees from the same planting on MM.111 EMLA and M.9 EMLA in all three training systems. Training system effects interacted with rootstock effects in dry weights of branches and of fruit. Nonpruned system shoot and fruit dry weights reflected known rootstock vigor; whereas, pruned system (three-wire and central leader) shoot dry weights were greatest and fruit dry weights were lowest in trees on M.7a. Rootstock affected the partitioning of dry matter between above- and below-ground tree components, with MM.111 EMLA accumulating significantly more dry matter in the root system than trees on the other rootstocks. Trees in the central leader and the three-wire palmette systems partitioned more dry weight into nonbearing 1-year shoots than trees in the nonpruned system. Root starch content at harvest was greater in trees on MM.111 EMLA than on M.9 EMLA, and root sucrose and sorbitol were less in trees on MM.111 EMLA compared to M.9 EMLA. At leaf fall, starch in young roots was equal in trees on both rootstocks, and sorbitol again was lower in trees on MM.111 EMLA. Harvest starch content of roots, shoots, and branches was lower in nonpruned than in pruned trees. At leaf fall, root, shoot, and branch starch content increased in nonpruned and central leader-trained trees but did not increase in three-wire palmette-trained trees.
\end{abstract}

Extensive studies with apple have shown the effects of various training systems and rootstocks on dry-matter and soluble carbohydrate composition of leaves, fruit, and spurs (Brown et al., 1985b; Drake et al., 1988; Ferree, 1989; Taylor and Ferree, 1986; Schechter et al., 1991). There are few published reports, however, that quantify the influence of either cultural practices or size-controlling rootstocks on the total partitioning of dry matter and soluble carbohydrates among reproductive, vegetative, and structural components of an apple tree. These data are needed to develop valid management models for commercial orchards.

Reich (1985) demonstrated that dormant pruning decreased trunk (bark and wood) starch accumulation but did not affect total fruit or shoot dry weight. Strong and Miller-Azarenko (1991) showed that dwarfing rootstocks of apple accumulated less branch and frame dry weight and greater fruit, spur, and spur leaf dry weight compared to rootstocks with moderate or low vigor control. Brown et al. (1985a) reported that apple trees on M.9 had less

Received for publication 4 June 1993. Accepted for publication 28 Sept. 1993. Approved for publication by the director of the West Virginia Agricultural and Forestry Experiment Station as scientific article no. 2417. This research was supported by Hatch funds, project no. 337. We also gratefully acknowledge support provided by the International Dwarf Fruit Tree Association and the West Virginia Tree Fruit Improvement Assessment Board. We thank Barton Baker, Suman Singha, Ed Townsend, Zhongchun Wang, Bill Bengar, Jim Wood, Timothy Winfield, Janice Stutte, and the experiment station farm staff for their valuable contributions toward this research. The cost of publishing this paper was defrayed in part by the payment of page charges. Under postal regulations, this paper therefore must be hereby marked advertisement solely to indicate this fact. above- and below-ground dry weight than trees on MM.111 and that carbohydrate content followed a similar pattern. Distribution was determined throughout a season, and differences between rootstocks were most apparent from harvest through leaf fall.

The objective of this study was to determine the effect of apple rootstocks and training systems on 1) dry weight distribution and 2) deposition of reserve and transport carbohydrates at harvest and during the dormant period. An experimental design was developed that integrated rootstock and training system with $\mathrm{C}$ partitioning and productive capacity.

\section{Materials and Methods}

'Golden Delicious (Gibson)' trees were planted in 1986 at the West Virginia Univ. Experiment Station, Kearneysville, in a randomized block, split-plot design comparing four rootstocks (MM.111 EMLA, M.7a, M.26 EMLA, and M.9 EMLA) and three training systems (three-wire palmette trellis, free-standing central leader, and nonpruned). There were 10 trees (replicates) per rootstock (split plots) in a given system (main plots). Tree spacing was $3 \times 3 \mathrm{~m}$. All trees were headed at $66 \mathrm{~cm}$ at planting. Trees on the three-wire palmette trellis were trained to the system described by Singha et al. (1990); trees in the free-standing central leader system were trained as described by Heinicke (1974); and trees in the nonpruned system were neither trained nor pruned other than the initial heading cut. The experimental planting was maintained according to current West Virginia Univ. Extension recommenda- 
tions (Hogmire et al., 1993). Freezing temperatures killed flowers in 1989 and 1990, and the first commercial crop was harvested in 1991.

Trees from five replicates were removed at fruit maturity in 1991 to determine dry-matter distribution and mobilizable carbohydrate levels. The remaining trees were sampled at leaf fall for additional carbohydrate analysis. Trees were excavated on 17 Sept. 1991 using a tractor-mounted stump puller (Deiner and Domigan, 1986). Additional root excavation by hand demonstrated that the stump puller consistently harvested $\approx 85 \%$ of the root mass (data not shown). Trees were air-dried, partitioned into trunk, root, 1-year shoots, and 2-year and older ( $\geq 2$-year) branches and leaf fractions, then weighed. Bark, young roots $(<1 \mathrm{~mm}$ in diameter), mature roots ( $\geq 1 \mathrm{~mm}$ in diameter), 1-year shoots, and 2-year branches, spurs, and leaves from M.9 EMLA and MM.111 EMLA rootstocks (all training systems) were subsampled on 18 Sept. from the excavated trees and on 24 Nov. from the replicates remaining in the field. The subsamples were cut into thin slices, lyophilized, and ground in a Wiley mill to pass through a 40-mesh screen.

Carbohydrates were analyzed using a modification of the method of Wang and Stutte (1992). Tissue $(0.5 \mathrm{~g})$ was extracted twice in $5 \mathrm{ml}$ boiling water and centrifuged. The pooled supernatant was passed through a polyvinyl-pyrrolidone column, and glucose, fructose, sucrose, and sorbitol were quantified using highperformance liquid chromatography and refractive-index spectroscopy. The pellet was resuspended in $10 \mathrm{ml}$ phosphate buffer with amyloglucosidase (10 mg EC 3.2.1.3/100 ml), vortexed, incubated at $55 \mathrm{C}$ in a water bath shaker for $16 \mathrm{~h}$, and centrifuged. The supernatant was assayed colorimetrically for conversion of starch to glucose.

Data were analyzed using the General Linear Model Procedure (GLM) of the Statistical Analysis System Institute (Cary, N.C.). Means were separated by least significant difference. An arcsin square-root transformation was performed on percent data.
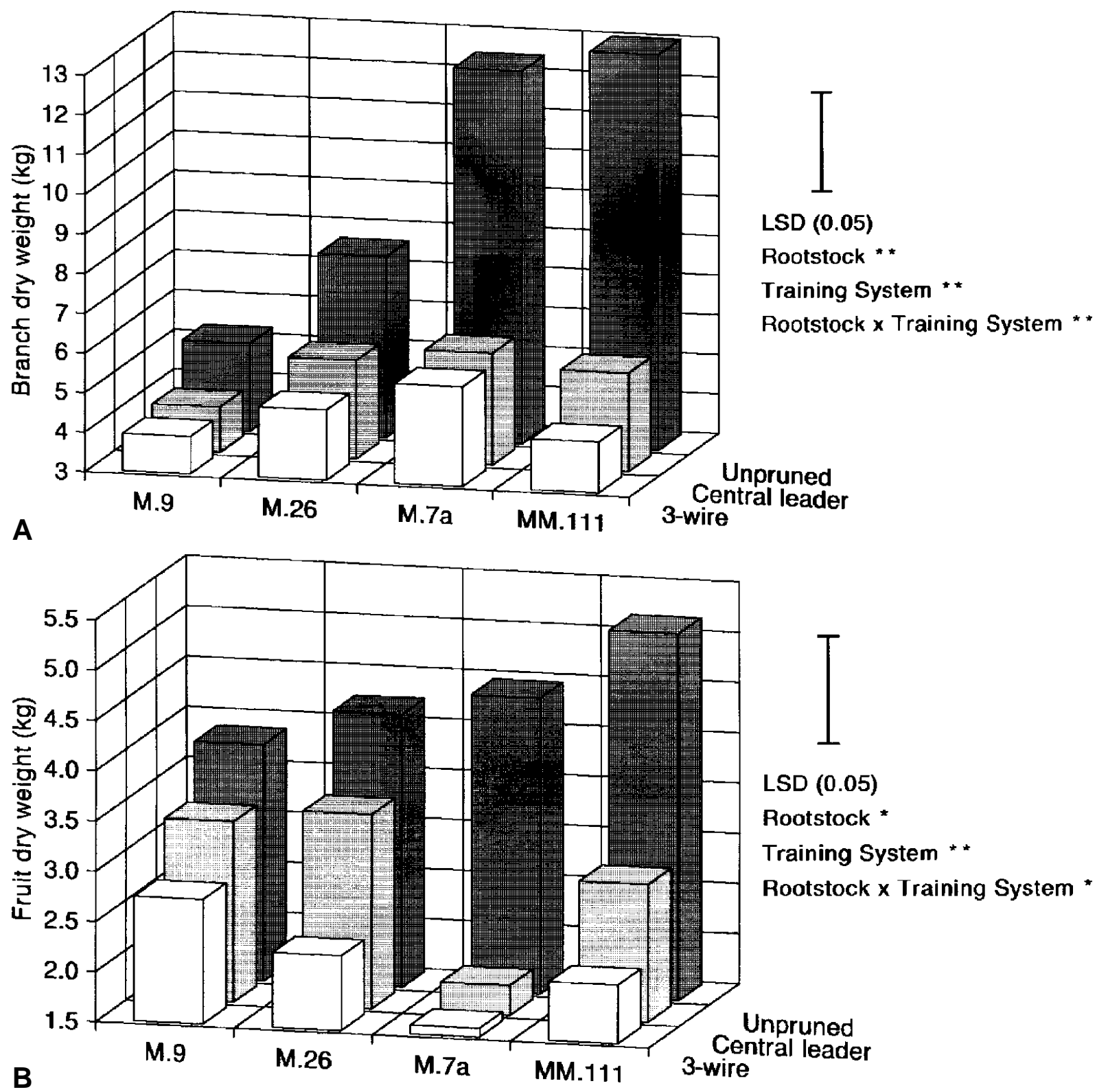

Fig. 1. Interaction of training system and rootstock on fruit (A) and 2-year branch (B) dry weight of 5-year-old 'Golden Delicious' apple trees excavated on 17 Sept. 1991 (five replicates, $\mathrm{n}=15$ ). 


\section{Results and Discussion}

The data collected reflect tree superstructure accumulated over six seasons and fruit, leaf, and carbohydrate dry weights for the sixth year of growth of 'Golden Delicious' trees. Rootstock and training-system interactions were found for dry weights of $\geq 2$-year branches and fruit, and these are discussed in Fig. 1 and the text. For all other comparisons, only rootstock and training-system main effects are presented.

Dry-matter distribution. Training system affected branch drymatter accumulation differently on different rootstocks (Fig. 1A). Trees trained to either a central-leader or three-wire palmette training system accumulated 50\% less dry matter in branches than nonpruned trees. Pruned trees grown on M.7a accumulated $46 \%$ more dry matter in branches than pruned trees on M.9 EMLA. Training system had more impact on branch biomass of trees grown on vigorous rootstocks (M.7a and MM.111 EMLA) than of trees on dwarfing rootstocks (M.9 EMLA and M.26 EMLA) because they required heavier pruning. Branch dry weight of nonpruned trees was directly proportional to rootstock vigor.

Training system also influenced productivity of trees on different rootstocks (Fig. 1B). Fruit dry weight in the nonpruned system was directly proportional to rootstock vigor. Pruned trees on M.7a had $23 \%$ less fruit dry weight than pruned trees on the other rootstocks. Yield (fruit dry weight) in the three-wire palmette and the central leader systems was strongly influenced by vegetative vigor (shoot dry weight) $(r=-0.88 ; P=0.05$; data not shown). New growth stimulated by pruning apparently reduced yields of trees on M.7a.

Rootstock affected dry-matter partitioning into above- and below-ground components of the apple tree (Table 1). Trees on MM.111 EMLA had 51\% greater absolute dry weight in roots than trees on M.9 EMLA. Root : shoot $(\mathrm{R}: \mathrm{S})$ ratios-root dry weight/ shoot + branch + trunk dry weights) - were calculated, and trees on MM.111 EMLA and M.26 EMLA had higher R : S ratios than trees on M.9 EMLA and M.7a. These data are consistent with those of Brown et al. (1985a), who reported that MM.111 partitioned more dry matter to the below-ground portions of a tree than M.9. Trees on M.9 EMLA partitioned less dry matter to structural components, e.g., branches, than M.7a or MM.111 EMLA. These results are consistent with those reported by Strong and Miller-Azarenko (1991). Total dry weights were proportional to rootstock vigor.

Training system also affected the distribution of dry matter among various apple tree tissues (Table 2). The nonpruned treatment had $65 \%$ less dry matter partitioned to 1-year-old shoots than the three-wire palmette. The nonpruned treatment also had the lowest percentage of dry matter partitioned into leaves and trunks. Nonpruned trees had the greatest absolute dry weight partitioned

Table 1. Influence of rootstock on dry-matter distribution in 'Golden Delicious' apple trees planted in 1986 and excavated 17 Sept. 1991.

\begin{tabular}{|c|c|c|c|c|c|c|c|}
\hline Rootstock & Shoots & Branches $^{y}$ & Leaves & Trunk & Roots & Fruit $^{\mathrm{x}}$ & \\
\hline \multicolumn{8}{|c|}{ Dry wt $(\mathrm{kg} /$ tree $)$} \\
\hline & & & & & & & Total \\
\hline M.9 EMLA & $0.69 \mathrm{~b}^{\mathrm{z}}$ & $4.41 \mathrm{c}$ & $1.35 \mathrm{~b}$ & $3.31 \mathrm{c}$ & $2.59 \mathrm{c}$ & $3.26 \mathrm{a}$ & $15.61 \mathrm{c}$ \\
\hline M.26 EMLA & $0.87 \mathrm{a}$ & $5.95 \mathrm{bc}$ & $1.50 \mathrm{~b}$ & $3.96 \mathrm{~b}$ & $4.38 \mathrm{~b}$ & $3.30 \mathrm{a}$ & $19.95 \mathrm{~b}$ \\
\hline M.7a & $1.02 \mathrm{a}$ & $7.92 \mathrm{a}$ & $1.85 \mathrm{a}$ & $4.82 \mathrm{a}$ & $4.57 \mathrm{~b}$ & $2.61 \mathrm{~b}$ & $22.78 \mathrm{a}$ \\
\hline MM.111 EMLA & $0.95 \mathrm{a}$ & $7.58 \mathrm{ab}$ & $1.65 \mathrm{ab}$ & $4.55 \mathrm{a}$ & $5.29 \mathrm{a}$ & $3.37 \mathrm{a}$ & $23.39 \mathrm{a}$ \\
\hline \multicolumn{8}{|c|}{ Dry-matter fractions ( $\%$ dry wt) } \\
\hline & & & & & & & $\mathrm{R}: \mathrm{S}^{\mathrm{w}}$ \\
\hline M.9 EMLA & $4.5 \mathrm{a}$ & $28.2 \mathrm{c}$ & $8.9 \mathrm{a}$ & $21.4 \mathrm{ab}$ & $16.3 \mathrm{~b}$ & $21.3 \mathrm{a}$ & $0.3 \mathrm{~b}$ \\
\hline M.26 EMLA & $4.5 \mathrm{a}$ & $29.4 \mathrm{bc}$ & $7.5 \mathrm{a}$ & $20.1 \mathrm{~b}$ & $22.0 \mathrm{a}$ & $16.3 \mathrm{a}$ & $0.4 \mathrm{a}$ \\
\hline M.7a & $4.8 \mathrm{a}$ & $33.1 \mathrm{a}$ & $8.2 \mathrm{a}$ & $22.5 \mathrm{a}$ & $20.8 \mathrm{a}$ & $10.6 \mathrm{~b}$ & $0.3 \mathrm{~b}$ \\
\hline MM.111 EMLA & $4.5 \mathrm{a}$ & $30.4 \mathrm{~b}$ & $7.3 \mathrm{a}$ & $20.5 \mathrm{ab}$ & $23.1 \mathrm{a}$ & $14.1 \mathrm{ab}$ & $0.4 \mathrm{a}$ \\
\hline
\end{tabular}

${ }^{\mathrm{z}}$ Mean separation in columns by LSD, $P \leq 0.05, \mathrm{n}=15$ (percentage data analyzed by arcsin square-root transformation; nontransformed data shown). y Significant training system $\times$ rootstock interaction; mean separation for pruned trees (Fig. 1A).

${ }^{\mathrm{x}}$ Significant training system $\times$ rootstock interaction; mean separation for pruned trees (Fig. 1B).

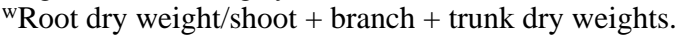

Table 2. Influence of training system on dry-matter distribution in 'Golden Delicious' apple trees planted in 1986 and excavated 17 Sept. 1991.

\begin{tabular}{|c|c|c|c|c|c|c|c|}
\hline Training system & Shoots & Branches $^{y}$ & Leaves & Trunk & Roots & Fruit $^{\mathrm{x}}$ & \\
\hline \multicolumn{8}{|c|}{ Dry wt $(\mathrm{kg} /$ tree $)$} \\
\hline & & & & & & & Total \\
\hline Three-wire & $1.29 \mathrm{a}^{\mathrm{z}}$ & $4.63 \mathrm{~b}$ & $1.47 \mathrm{a}$ & $3.73 \mathrm{~b}$ & $3.66 \mathrm{~b}$ & $2.17 \mathrm{c}$ & $16.93 \mathrm{~b}$ \\
\hline Central leader & $0.77 \mathrm{~b}$ & $5.35 \mathrm{~b}$ & $1.60 \mathrm{a}$ & $4.52 \mathrm{a}$ & $4.05 \mathrm{~b}$ & $2.83 \mathrm{~b}$ & $19.11 \mathrm{~b}$ \\
\hline \multirow[t]{3}{*}{ Nonpruned } & $0.58 \mathrm{c}$ & $9.73 \mathrm{a}$ & $1.73 \mathrm{a}$ & $4.34 \mathrm{a}$ & $5.12 \mathrm{a}$ & $4.45 \mathrm{a}$ & $25.96 \mathrm{a}$ \\
\hline & & & Dry-matter & $\%$ dry $\mathrm{n}$ & & & \\
\hline & & & & & & & $\mathrm{R}: \mathrm{S}^{\mathrm{w}}$ \\
\hline Three-wire & $7.5 \mathrm{a}$ & $27.2 \mathrm{ab}$ & $8.7 \mathrm{a}$ & $22.1 \mathrm{a}$ & $21.3 \mathrm{a}$ & $13.3 \mathrm{a}$ & $0.4 \mathrm{a}$ \\
\hline Central leader & $4.0 \mathrm{~b}$ & $27.7 \mathrm{~b}$ & $8.4 \mathrm{ab}$ & $24.0 \mathrm{a}$ & $21.1 \mathrm{a}$ & $15.1 \mathrm{a}$ & $0.4 \mathrm{a}$ \\
\hline Nonpruned & $2.2 \mathrm{c}$ & $36.3 \mathrm{a}$ & $6.7 \mathrm{~b}$ & $17.4 \mathrm{~b}$ & $19.8 \mathrm{a}$ & $17.9 \mathrm{a}$ & $0.4 \mathrm{a}$ \\
\hline
\end{tabular}

${ }^{\mathrm{z}}$ Mean separation in columns by LSD, $P \leq 0.05, \mathrm{n}=20$ (percentage data analyzed by arcsin square-root transformation; nontransformed data shown). ${ }^{y}$ Significant training system $\times$ rootstock interaction (Fig. 1A).

${ }^{x}$ Significant training system $\times$ rootstock interaction (Fig. 1B).

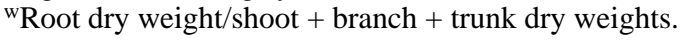


Table 3. Influence of rootstock and training system on C conversion index $(\mathrm{F}: \mathrm{L})$ of 'Golden Delicious' apple trees planted in 1986 and excavated 17 Sept. 1991.

\begin{tabular}{|c|c|}
\hline Influence & $F: L^{y}$ \\
\hline \multicolumn{2}{|c|}{ Rootstock } \\
\hline M.9 EMLA & $2.5 \mathrm{a}^{\mathrm{z}}$ \\
\hline M.26 EMLA & $2.2 \mathrm{ab}$ \\
\hline M.7a & $1.4 \mathrm{c}$ \\
\hline MM.111 EMLA & $2.0 \mathrm{~b}$ \\
\hline \multicolumn{2}{|c|}{ Training system } \\
\hline Three-wire & $1.6 \mathrm{~b}$ \\
\hline Central leader & $1.8 \mathrm{~b}$ \\
\hline Nonpruned & $2.7 \mathrm{a}$ \\
\hline
\end{tabular}

${ }^{\mathrm{z}}$ Mean separation in columns by LSD, $P \leq 0.05, \mathrm{n}=20$ for rootstocks, $\mathrm{n}=15$ for systems (data analyzed by arcsin square-root transformation; nontransformed data shown).

${ }^{\mathrm{y}}$ Fruit dry weight/leaf dry weight. into branches, roots, and fruit, but percentage of dry matter in these fractions was similar to that in the other training systems. The three-wire palmette, free-standing central leader, and nonpruned trees had comparable $\mathrm{R}: \mathrm{S}$ values.

Leaves are the ultimate source of all $\mathrm{C}$ partitioned into fruit, and the relative efficiencies of the rootstocks and training systems at partitioning fixed $\mathrm{C}$ to fruit can be considered to be a function of the leaf. An index of $\mathrm{C}$ conversion can be expressed as fruit dry weight per tree per leaf dry weight per tree (Charles-Edwards, 1982). When systems were compared during the sixth year using this fruit : leaf $(\mathrm{F}: \mathrm{L})$ ratio, the value for no pruning exceeded the values for central leader and three-wire palmette training by $44 \%$ and $41 \%$, respectively (Table 3 ). Rootstock also affected $\mathrm{F}$ : L ratio. Trees on M.7a had a lower F : L ratio than trees on the other rootstocks, a result that seemed to be related to competition between vegetative and reproductive sinks in the three-wire palmette and central leader systems.

Table 4. Influence of rootstock on the carbohydrate (CHO) fractions of 5-year-old 'Golden Delicious' apple trees sampled at two growth stages.

\begin{tabular}{|c|c|c|c|c|}
\hline \multirow{3}{*}{$\begin{array}{l}\text { CHO } \\
\text { fraction } \\
\text { Starch }\end{array}$} & \multirow{2}{*}{$\begin{array}{c}\text { Plant } \\
\text { part }\end{array}$} & \multirow[b]{2}{*}{ Rootstock } & Harvest & Leaf fall \\
\hline & & & \multicolumn{2}{|c|}{$\left(\mathrm{mg} \cdot \mathrm{g}^{-1}\right.$ dry wt $)$} \\
\hline & Young roots & M.9 EMLA & $91.3 \mathrm{~b}^{2}$ & $151.6 \mathrm{a}$ \\
\hline & & MM.111 EMLA & $129.5 \mathrm{a}$ & $163.9 \mathrm{a}$ \\
\hline & Mature roots & M.9 EMLA & $123.1 \mathrm{~b}$ & --- \\
\hline & & MM.111 EMLA & $153.2 \mathrm{a}$ & --- \\
\hline & Leaves & M.9 EMLA & $6.5 \mathrm{a}$ & --- \\
\hline & & MM.111 EMLA & $6.4 \mathrm{a}$ & --- \\
\hline & Spurs & M.9 EMLA & $6.3 \mathrm{a}$ & --- \\
\hline & & MM.111 EMLA & $6.0 \mathrm{a}$ & --- \\
\hline & Shoots & M.9 EMLA & $42.3 \mathrm{a}$ & $46.7 \mathrm{~b}$ \\
\hline & & MM.111 EMLA & $43.7 \mathrm{a}$ & $51.1 \mathrm{a}$ \\
\hline & Branches & M.9 EMLA & $33.9 \mathrm{a}$ & $37.9 \mathrm{~b}$ \\
\hline & & MM.111 EMLA & $34.9 \mathrm{a}$ & $48.1 \mathrm{a}$ \\
\hline & Bark (trunk) & M.9 EMLA & $29.3 \mathrm{a}$ & --- \\
\hline & & MM.111 EMLA & $30.9 \mathrm{a}$ & --- \\
\hline \multirow[t]{14}{*}{ Sucrose } & Young roots & M.9 EMLA & $21.7 \mathrm{a}$ & $96.6 \mathrm{a}$ \\
\hline & & MM.111 EMLA & $16.5 \mathrm{~b}$ & $41.0 \mathrm{a}$ \\
\hline & Mature roots & M.9 EMLA & $14.2 \mathrm{a}$ & --- \\
\hline & & MM.111 EMLA & $11.2 \mathrm{a}$ & --- \\
\hline & Leaves & M.9 EMLA & $95.7 \mathrm{a}$ & --- \\
\hline & & MM.111 EMLA & $114.3 \mathrm{a}$ & --- \\
\hline & Spurs & M.9 EMLA & $101.5 \mathrm{a}$ & --- \\
\hline & & MM.111 EMLA & $128.6 \mathrm{a}$ & --- \\
\hline & Shoots & M.9 EMLA & $17.1 \mathrm{a}$ & $131.9 \mathrm{a}$ \\
\hline & & MM.111 EMLA & $15.8 \mathrm{a}$ & $97.0 \mathrm{a}$ \\
\hline & Branches & M.9 EMLA & $13.8 \mathrm{a}$ & $144.7 \mathrm{a}$ \\
\hline & & MM.111 EMLA & $14.5 \mathrm{a}$ & $95.9 \mathrm{a}$ \\
\hline & Bark (trunk) & M.9 EMLA & $27.0 \mathrm{a}$ & --- \\
\hline & & MM.111 EMLA & $17.7 \mathrm{a}$ & --- \\
\hline \multirow[t]{14}{*}{ Sorbitol } & Young roots & M.9 EMLA & $30.7 \mathrm{a}$ & $19.0 \mathrm{a}$ \\
\hline & & MM.111 EMLA & $20.3 \mathrm{~b}$ & $6.1 \mathrm{~b}$ \\
\hline & Mature roots & M.9 EMLA & $21.9 \mathrm{a}$ & --- \\
\hline & & MM.111 EMLA & $9.9 \mathrm{~b}$ & --- \\
\hline & Leaves & M.9 EMLA & $144.0 \mathrm{a}$ & --- \\
\hline & & MM.111 EMLA & $226.8 \mathrm{a}$ & --- \\
\hline & Spurs & M.9 EMLA & $133.3 \mathrm{a}$ & --- \\
\hline & & MM.111 EMLA & $184.2 \mathrm{a}$ & --- \\
\hline & Shoots & M.9 EMLA & $26.4 \mathrm{a}$ & $80.5 \mathrm{a}$ \\
\hline & & MM.111 EMLA & $26.3 \mathrm{a}$ & $74.1 \mathrm{a}$ \\
\hline & Branches & M.9 EMLA & $20.0 \mathrm{a}$ & $54.7 \mathrm{a}$ \\
\hline & & MM.111 EMLA & $22.8 \mathrm{a}$ & $46.0 \mathrm{a}$ \\
\hline & Bark (trunk) & M.9 EMLA & $49.1 \mathrm{a}$ & --- \\
\hline & & MM.111 EMLA & $36.6 \mathrm{a}$ & --- \\
\hline
\end{tabular}

${ }^{\mathrm{z}}$ Mean separation in columns, CHO fractions, and plant parts by LSD, $P \leq 0.05, \mathrm{n}=5$, system $\times$ rootstock interaction nonsignificant. 
Table 5. Influence of training system on the carbohydrate (CHO) fractions of 5-year-old 'Golden Delicious' apple trees sampled at two growth stages.

\begin{tabular}{|c|c|c|c|c|}
\hline \multirow{2}{*}{$\begin{array}{l}\mathrm{CHO} \\
\text { fraction }\end{array}$} & \multirow{2}{*}{$\begin{array}{c}\text { Plant } \\
\text { part }\end{array}$} & \multirow{2}{*}{$\begin{array}{l}\text { Training } \\
\text { system }\end{array}$} & Harvest & Leaf fall \\
\hline & & & \multicolumn{2}{|c|}{$\left(\mathrm{mg} \cdot \mathrm{g}^{-1}\right.$ dry wt) } \\
\hline \multirow[t]{21}{*}{$\overline{\text { Starch }}$} & Young roots & Three-wire & $110.1 b^{z}$ & $120.4 \mathrm{~b}$ \\
\hline & & Central leader & $144.4 \mathrm{a}$ & $184.3 \mathrm{a}$ \\
\hline & & Nonpruned & $85.7 \mathrm{c}$ & $167.0 \mathrm{a}$ \\
\hline & Mature roots & Three-wire & $147.5 \mathrm{a}$ & --- \\
\hline & & Central leader & $158.7 \mathrm{a}$ & --- \\
\hline & & Nonpruned & $118.5 \mathrm{~b}$ & --- \\
\hline & Leaves & Three-wire & $7.0 \mathrm{a}$ & --- \\
\hline & & Central leader & $6.3 \mathrm{ab}$ & --- \\
\hline & & Nonpruned & $5.8 \mathrm{~b}$ & --- \\
\hline & Spurs & Three-wire & $6.5 \mathrm{a}$ & --- \\
\hline & & Central leader & $6.0 \mathrm{a}$ & --- \\
\hline & & Nonpruned & $5.9 \mathrm{a}$ & --- \\
\hline & Shoots & Three-wire & $49.0 \mathrm{a}$ & $48.9 \mathrm{ab}$ \\
\hline & & Central leader & $45.5 \mathrm{a}$ & $52.7 \mathrm{a}$ \\
\hline & & Nonpruned & $31.5 \mathrm{~b}$ & $45.3 \mathrm{~b}$ \\
\hline & Branches & Three-wire & $38.1 \mathrm{a}$ & $39.4 \mathrm{~b}$ \\
\hline & & Central leader & $36.2 \mathrm{a}$ & $49.0 \mathrm{a}$ \\
\hline & & Nonpruned & $26.9 \mathrm{~b}$ & $42.4 \mathrm{~b}$ \\
\hline & Bark (trunk) & Three-wire & $30.1 \mathrm{a}$ & --- \\
\hline & & Central leader & $30.3 \mathrm{a}$ & --- \\
\hline & & Nonpruned & $29.9 \mathrm{a}$ & --- \\
\hline \multirow[t]{21}{*}{ Sucrose } & Young roots & Three-wire & $21.9 \mathrm{a}$ & $11.8 \mathrm{~b}$ \\
\hline & & Central leader & $19.2 \mathrm{a}$ & $113.5 \mathrm{a}$ \\
\hline & & Nonpruned & $12.8 \mathrm{~b}$ & $81.2 \mathrm{ab}$ \\
\hline & Mature roots & Three-wire & $13.9 \mathrm{a}$ & --- \\
\hline & & Central leader & $11.4 \mathrm{a}$ & --- \\
\hline & & Nonpruned & $11.8 \mathrm{a}$ & --- \\
\hline & Leaves & Three-wire & $48.0 \mathrm{a}$ & --- \\
\hline & & Central leader & $93.3 \mathrm{a}$ & --- \\
\hline & & Nonpruned & $161.3 \mathrm{a}$ & --- \\
\hline & Spurs & Three-wire & $94.0 \mathrm{a}$ & --- \\
\hline & & Central leader & $124.5 \mathrm{a}$ & --- \\
\hline & & Nonpruned & $128.6 \mathrm{a}$ & --- \\
\hline & Shoots & Three-wire & $19.3 \mathrm{a}$ & $48.8 \mathrm{a}$ \\
\hline & & Central leader & $13.3 \mathrm{~b}$ & $140.0 \mathrm{a}$ \\
\hline & & Nonpruned & $16.1 \mathrm{ab}$ & $153.5 \mathrm{a}$ \\
\hline & Branches & Three-wire & $12.1 \mathrm{~b}$ & $22.7 \mathrm{~b}$ \\
\hline & & Central leader & $15.1 \mathrm{a}$ & $153.6 \mathrm{ab}$ \\
\hline & & Nonpruned & $15.8 \mathrm{a}$ & $175.8 \mathrm{a}$ \\
\hline & Bark (trunk) & Three-wire & $20.1 \mathrm{a}$ & --- \\
\hline & & Central leader & $17.7 \mathrm{a}$ & --- \\
\hline & & Nonpruned & $28.7 \mathrm{a}$ & --- \\
\hline \multirow[t]{21}{*}{ Sorbitol } & Young roots & Three-wire & $27.5 \mathrm{a}$ & $6.6 \mathrm{~b}$ \\
\hline & & Central leader & $23.2 \mathrm{a}$ & $19.6 \mathrm{a}$ \\
\hline & & Nonpruned & $20.8 \mathrm{a}$ & $10.6 \mathrm{~b}$ \\
\hline & Mature roots & Three-wire & $15.7 \mathrm{a}$ & --- \\
\hline & & Central leader & $13.0 \mathrm{a}$ & --- \\
\hline & & Nonpruned & $15.8 \mathrm{a}$ & --- \\
\hline & Leaves & Three-wire & $119.2 \mathrm{a}$ & --- \\
\hline & & Central leader & $177.7 \mathrm{a}$ & --- \\
\hline & & Nonpruned & $260.4 \mathrm{a}$ & --- \\
\hline & Spurs & Three-wire & $149.1 \mathrm{a}$ & --- \\
\hline & & Central leader & $175.7 \mathrm{a}$ & --- \\
\hline & & Nonpruned & $169.9 \mathrm{a}$ & --- \\
\hline & Shoots & Three-wire & $23.6 \mathrm{~b}$ & $63.4 \mathrm{a}$ \\
\hline & & Central leader & $29.7 \mathrm{a}$ & $85.2 \mathrm{a}$ \\
\hline & & Nonpruned & $25.9 \mathrm{~b}$ & $83.5 \mathrm{a}$ \\
\hline & Branches & Three-wire & $16.2 \mathrm{c}$ & $15.3 \mathrm{~b}$ \\
\hline & & Central leader & $21.4 \mathrm{~b}$ & $57.7 \mathrm{ab}$ \\
\hline & & Nonpruned & $29.0 \mathrm{a}$ & $76.6 \mathrm{a}$ \\
\hline & Bark (trunk) & Three-wire & $36.7 \mathrm{a}$ & --- \\
\hline & & Central leader & $37.4 \mathrm{a}$ & --- \\
\hline & & Nonpruned & $56.0 \mathrm{a}$ & --- \\
\hline
\end{tabular}

${ }^{\mathrm{z}}$ Mean separation within columns, CHO fractions, and plant parts by LSD, $P \leq 0.05, \mathrm{n}=5$, rootstock $\times$ system interaction nonsignificant. 
Mobilizable carbohydrate levels. Training system and rootstock also affected the concentration of mobilizable carbohydrates in 'Golden Delicious' apple trees. These data are shown in Tables 4 and 5. Glucose and fructose concentrations paralleled sucrose contents, and the data are not shown.

Rootstock affected concentrations of starch, sucrose, and sorbitol at harvest and concentrations of starch and sorbitol at leaf fall. Trees grown on the more-vigorous MM.111 EMLA rootstock had greater root starch and less root sorbitol and sucrose at harvest than trees on the less-vigorous M.9 EMLA rootstock (Table 4). Starch content of young and mature roots in trees on MM.111 EMLA was $29 \%$ and $20 \%$ higher, respectively, than in trees on M.9 EMLA. Sorbitol content was $44 \%$ and $55 \%$ lower, respectively, and sucrose content of young roots was $24 \%$ lower than in trees on M.9 EMLA. Trees grown on MM.111 EMLA had greater shoot and branch starch (8\% and $9 \%$, respectively) and less root sorbitol and sucrose at leaf fall (69\% and 58\%, respectively). Root starch in M.9 EMLA increased by $40 \%(P \leq 0.05)$ at leaf fall. These findings confirm previous reports that trees on more vigorous rootstocks have a higher starch content than trees on dwarfing rootstocks (Brown et al., 1985; Gaudillere et al., 1992). The results also agree with previous reports indicating an inverse relationship between a high rate of shoot growth and root sorbitol and sucrose content (Brown et al., 1985).

Training system affected starch, sucrose, and sorbitol content at harvest and leaf fall. Nonpruned trees had lower root, leaf, shoot, and branch starch and lower young root sucrose but greater branch sorbitol concentration at harvest than pruned trees (Table 5). Starch content of young roots was $25 \%$ higher in 'Golden Delicious' trained to the central leader than in trees trained to the threewire palmette and $40 \%$ higher than in nonpruned trees. Starch content of mature roots of trees trained to the central leader and the three-wire palmette systems averaged $29 \%$ higher than that of nonpruned trees. Leaf, shoot, and branch starch content was $10 \%$, $35 \%$, and $25 \%$ lower, respectively, in nonpruned vs. pruned systems, and young root sucrose was 35\% lower. After harvest, starch concentration in young roots and shoots and branches increased (49\%, 31\%, and $46 \%$, respectively) in nonpruned trees but did not increase in three-wire palmette-trained trees $(P \leq 0.05)$. Sucrose concentration in young roots and branches increased in nonpruned trees at leaf fall but not in three-wire palmette-trained trees $(P \leq 0.05)$. Sorbitol content of young roots in nonpruned and palmette-trained trees at leaf fall was less than that of central leader-trained trees. As reported by Reich (1985), the greatest effect of pruning was on distribution to energy reserves in the form of starch. The effects on sucrose and sorbitol need to be explored further.

This research provides data that can be used to define further the effects of rootstock and training system on $\mathrm{C}$ use in apple. Training method interacted with rootstock to affect dry-matter partitioning in bearing, field-grown apple trees. Fruit dry weight was proportional to tree vigor in the nonpruned system, whereas fruit dry weight was inversely proportional to tree vigor in the pruned systems. Where no interactions occurred, training and rootstock had distinct effects on $\mathrm{C}$ allocation. Pruning increased shoot dry weight and decreased branch, root, and fruit dry weight but did not affect overall $\mathrm{C}$ partitioning between above- and below-ground components. In contrast, size-controlling rootstocks affected drymatter partitioning between above- and below-ground components. Trees on M.9 EMLA and M.26 EMLA rootstocks and in the nonpruned system were most efficient at partitioning fixed $\mathrm{C}$ to fruit. Training system and rootstock influenced partitioning of starch, sorbitol, and sucrose. Pruning increased above- and belowground starch reserves at harvest and decreased branch sorbitol. At leaf fall, root and shoot starch and root sucrose increased in nonpruned and central leader-trained trees but showed no increase in three-wire palmette-trained trees. Rootstock effects at harvest seemed to be related to total dry weights of the trees, with MM.111 EMLA allocating $C$ to root starch and M.9 EMLA allocating $C$ to root sorbitol and sucrose. At leaf fall, M. 9 EMLA reallocated $\mathrm{C}$ to starch.

These carbohydrate data are consistent with relative cropping efficiencies of training systems and rootstocks. Trees on M.9 EMLA rootstock allocated dry matter and carbohydrates similarly to nonpruned trees, with both treatments resulting in large leaf and fruit sinks and corresponding reductions in reserves accumulated during the growing season. Root starch remained low through harvest and did not increase until leaf fall. The palmette trellis created a more compact canopy, but excessive pruning of trees on more-vigorous rootstocks resulted in increased shoot growth and decreased carbohydrates at leaf fall.

\section{Literature Cited}

Brown, C.S., E. Young, and D.M. Pharr. 1985a. Rootstock and scion effects on the seasonal distribution of dry weight and carbohydrates in young apple trees. J. Amer. Soc. Hort. Sci. 110:696-701.

Brown, C.S., E. Young, and D.M. Pharr. 1985b. Rootstock and scion effects on carbon partitioning in apple leaves. J. Amer. Soc. Hort. Sci. 110:701-705.

Charles-Edwards, D.A. 1982. Physiological determinants of crop growth. Academic Press, Sydney, Australia.

Deiner, R.G. and I. Domigan. 1986. Stump puller designed by WVU researchers. The Mountaineer Grower 458:12-14.

Drake, S.R., F.E. Larsen, J.K. Fellman, and S.S. Higgins. 1988. Maturity, storage quality, carbohydrate, and mineral content of 'Goldspur' apples as influenced by rootstock. J. Amer. Soc. Hort. Sci. 113:949-952.

Ferree, D.C. 1989. Influence of orchard management systems on spur quality, light, and fruit within the canopy of 'Golden Delicious' apple trees. J. Amer. Soc. Hort. Sci. 114:869-875.

Gaudillere, J., A. Moing, and F. Carbonne. 1992. Vigour and non-structural carbohydrates in young prune trees. Scientia Hort. 51:197-211.

Heinicke, D.R. 1974. High-density apple orchards-Planning, training and pruning. U.S. Dept. of Agr.-Agr. Res. Serv. Agr. Hdbk. 458.

Hogmire, H. W., A. Biggs, T. Baugher, M. Ingle, J. Kotcon, D. Monks, D. Pfeifer, R. Marini, R. Byers, R. Horsburgh, K. Yoder, R. Fell, J. Barden, M. Kushad, J. Derr, C. Foy, and M. Weaver. 1993. Virginia/West Virginia 1993 spray bulletin for commercial tree fruit growers. VPI/ WVU Publ. 456-419.

Reich, L. 1985. Carbohydrates in the apple tree: Effects associated with pruning and deblossoming. HortScience 20:238-240.

Schechter, I., D.C. Elfving, and J.T.A. Proctor. 1991. Rootstock affects vegetative growth characteristics and productivity of 'Delicious' apple. HortScience 26:1145-1148.

Singha, S., T.A. Baugher, and S.H. Blizzard. 1990. Intensive orcharding, a total system approach-3-wire trellis. West Virginia Univ. Coop. Ext. Serv. Circ. OM107.

Strong, D. and A. Miller-Azarenko. 1991. Dry matter partitioning in 'Starkspur Supreme Delicious' on nine rootstocks. Fruit Var. J. 45:238241.

Taylor, B.H. and D.C. Ferree. 1986. The influence of summer pruning and fruit cropping on the carbohydrate, nitrogen, and nutrient composition of apple trees. J. Amer. Soc. Hort. Sci. 111:342-346.

Wang, Z. and G.W. Stutte. 1992. The role of carbohydrates in active osmotic adjustment in apple under water stress. J. Amer. Soc. Hort. Sci. 117:816-823. 